\title{
Research on Phishing AP Attack Detection Technology Based on RSSI
}

\author{
Ling Jie \\ Faculty of Computer, Guangdong University \\ of Technology \\ Guangzhou, Guangdong, China \\ jling@gdut.edu.cn
}

\author{
Jin Shuangqi \\ Faculty of computer, Guangdong University \\ of Technology \\ Guangzhou, Guangdong, China \\ jsqfengbao@qq.com
}

\begin{abstract}
Wi-Fi wireless technology obtained rapid development because of its flexibility, mobility and easy scalability characteristics. But the network identifier (SSID,BSSID) is easy to be forged, the attacker is easy to forge AP that a common user can't identify it, and conduct other attacks, then get the user's sensitive information. In this paper, we propose an improved RSSI phishing AP attack detection method based on the characteristics of RSSI in wireless Wi-Fi network and easy to obtain. The method needs to be in a safe and secure environment construction of Wi-Fi wireless RSSI fingerprint database and the detected target $\mathrm{AP}$ and fingerprint library information were compared to determine the safety, solving the problem of twin fishing AP successfully. Experimental results show that in the RSSI threshold with the range of $-5 \mathrm{dBm}$, the correct detection rate can reach $95 \%$, to achieve the legitimacy of the WLAN AP detection.
\end{abstract}

\section{Keywords-Phishing AP Attack; WIFI; WLAN; RSSI}

\section{INTRODUCTION}

Wireless Local Area Network (WLAN) technology, according for its installation flexibility, mobility, easy to expand and get rapid development, with 3G / 4G technologies mature and popularization, based on 802.11 WIFI wireless network in bandwidth, coverage range obtained rapid increase has been carrying campus wireless, wireless medical, wireless city, wireless location, car wireless rich wireless applications, has gradually become a mainstream application market.

Wireless WI-FI brings convenience to people's work and life, but also has a variety of network attacks, with a lot of security risks. Wireless WIFI in communication between workstations is transmitted in the form of electromagnetic wave, any receiving device between the two stations can receive the data transmitted by the wireless local area network, a malicious user can tamper with the received data or other malicious operation. In a variety of wireless attacks, the fishing wireless AP attack is one of the most serious attacks harm, an attacker in a public place to build a camouflage of wireless AP, with identical to the real AP service set identifier (SSID) ${ }^{[1]}$, MAC address, encryption mode setting information, to lure victims connected to fake AP, and further to obtain user account passwords and other sensitive information ${ }^{[2-4]}$. Some foreign scholars are also called "Evil Twin AP" or "Rogue AP".

Traditional fishing AP detection technology, mainly on the server according to the wireless sniffer wireless monitoring network to detect Rogue AP, the sniffer can in the $2.4 \mathrm{GHz}$ and
$5 \mathrm{GHz}$ spectrum scanning of unauthorized network traffic, sniffing out the illegal network traffic. Some commercial detection products mainly on the use of this technology, such as Venus day clear wireless security engine, Motorola airdefense products. There are a number of products, using the characteristic fingerprint to distinguish between legitimate AP and phishing AP, these features include: MAC address, supplier name, signal strength, radio frequency measurement and SSID, etc. Other alternative methods also include the collection of RSSI, radio frequency changes and clock bias as a feature fingerprint to identify wireless phishing $\mathrm{AP}^{[5]}$.

In the paper [8] using the method of MAC address. In various WLAN, MAC address is distinguished for different network devices. The same data frame that is sent to the workstation in the wireless AP includes the MAC address information of the AP itself and the MAC address of the receiving party. And open the ESSID authentication AP will broadcast contains its own MAC address information of the beacon frame. In AP radio range station can receive this kind of frame, so an attacker can also obtain legitimate AP MAC address information and copy of these acquired Mac to illegal AP data frames. Because the MAC address can be easily changed by the attacker, so the use of MAC address detection, there are a lot of drawbacks.

Bratus in paper [9] proposed a kind of active detection method based on fingerprint. This method is by sending some specific error format of data frames to stimulate the wireless AP and different behavior characteristics through the detection of wireless AP to obtain for wireless AP chip card (chipset) or wireless network card driver (driver) and the characteristics of fingerprint information to distinguish between fishing wireless AP and legally authorized AP. Provides a detection method of this method with low cost to deploy wireless terminal active, namely through the modification of the data link layer data frame (MAC frame) and that some do not meet the provisions of the 802.11 standard protocol data frame in ${ }^{[10]}$, but not explicitly prohibited, so if a standard protocol clearly prohibit the case, the data frame may be discarded by AP, such as in the frame MAC management frame frame control (Frame Control) domain, which will be the two bit set to "1" and can create a malformed "stimulus" frame, the FromDS and ToDS fields in accordance with the agreement requirements should be set to "0", it can stimulate the AP, which made some of the characteristics of stimulus response behavior, different wireless LAN chip or wireless network card driver AP showed 
different behavior characteristics Is not the same, in addition to the literature ${ }^{[5]}$ also proposed the use of the structure of the decision tree to achieve automated response to the stimulus response. These detection methods based on AP fingerprint features can detect phishing AP, but these active detection methods are easily found by the attacker, and in the service side of the detection.

Pang in paper [11] discussed the IEEE802.11 protocol on hidden identifiers, highlighted the IEEE802.11 protocol in some unique identifier, the user is not anonymous, allows users to keep track of. Even if the associated identifier is deliberately obscured, it can be traced to the user by detecting the parameter information in a series of 802.11 protocols. 4 related network parameters are identified in this paper: the network destination address, the network name (SSID), the 802.11 protocol configuration options, and the size of the broadcast frame in the 802.11 protocol. In the case of encrypted traffic, 3/4 of the parameter information can still be acquired to. But in this kind of fingerprint recognition technology needs each customer end to last at least one hour the traffic sample monitoring, the monitoring event is long and the monitoring cost high.

Current fishing AP detection technology based on fingerprint feature need to establish a fingerprint database firstly, and this requires a large amount of overhead, and extract the fingerprint of the time is very long, so the real-time detection is relatively poor. However, the existing methods on the client are assumed to be a valid AP, but the different network types, bandwidth and congestion are different for each wireless network. This detection method also involves a flow regulation to eliminate noise interference. Since the client's detection technology is mostly based on statistical data, or based on the detection of wireless traffic in the 802.11 protocol in the distributed coordination function, the attacker needs to adjust the communication flow through.

\section{PHISHING AP ATTACK PRINCIPLE ANALYSIS}

An attacker can easily set up a fishing AP. First of all, we can build a wireless AP through some analysis software in the notebook computer. Then, the attacker use the configuration and legitimate AP the same MAC, SSID address, channel and encryption methods, and finally, waiting or to lure legitimate users to connect fishing AP. According to the IEEE 802.11 protocol standard, usually fishing wireless AP closer to the user more likely to succeed, when around multiple configurations of the same AP and wireless client will according to the wireless NIC select the AP with the strongest signal connection ${ }^{[6]}$. Therefore, as long as the attacker with the configuration and method the same AP information and improve signal strength and distance victims closer the easier it is to achieve the effect of the attack.

Attackers often in close proximity to shopping malls, hotels, cafes or in the library to build fishing AP, through fishing AP, an attacker can launched by the man in the middle attack to capture the user's sensitive information, such as account numbers, passwords and other, can also be through the operation of the DNS server, control circuit, launched more server phishing attacks. In a word, the fishing wireless AP serious harm to the security of WLAN.

Wireless phishing AP attack the basic step is that first attacker to pick a large signal power transmission AP; second, the attacker to obtain the legitimate SSID AP name, wireless channel, wireless encryption methods, such as configuration information. Finally the attacker to deploy a good AP wait for the user connection, or an attacker to send a message to deceive the initiative to AP, forcing the user to disconnect and legally authorized AP connection, wireless phishing AP attack scene graph as shown in figure 1 .

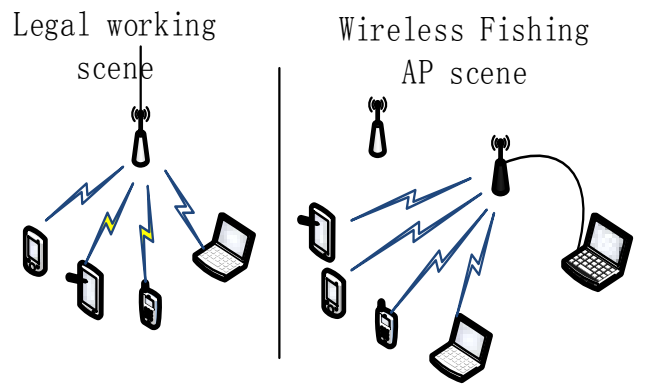

Fig. 1 legal scenes and wireless fishing scenes

At present, there are two types of fishing AP attack ${ }^{[7]}$, the first type is using typical wireless router as the fishing wireless AP, or through the refresh the wireless router firmware class establish AP special for fishing. Common third-party open source firmware DD-WRT and open wrt. The second type is in a portable notebook configuration two wireless card, a block is used to connect the real AP, so that the data transmitted to the Internet and other network card using the AP mode, configured to provide a wireless access AP, which is the famous fishing software radio AirSnarf (http://airsnarf.shmoo.com/). It is a simple malicious wireless access point settings using the program, designed to showcase a wireless fishing AP how to steal usernames and passwords from public wireless hotspots, AirSnarf is a cross platform software, now support Windows XP, Linux operating system, and can be used as firmware brush into the wireless router Linksys WRT54G series, and it also need other auxiliary automatic response tool to form a wireless fishing AP complete, such as DNS, WEB application service , dynamic web site environment etc.

\section{FISHING AP DETECTION METHOD BASED ON RSSI}

Received Signal Strength Value (RSSI) is used to identify the signal intensity. In the complex environment of the scene, the value of RSSI is not only influenced by the distance factors, but also because the effect of reflection, multipath effect, obstacle factors. According to the signal attenuation formula (1):

$$
\text { RSSI }=-(10 n \lg d+A)
$$

From the formula (1), we can know that the signal receiving strength value RSSI is affected by the variable A and variable $n, A$ is the signal strength value when the distance AP position is $\mathrm{n}, 1 \mathrm{~m}$ is the wireless signal transmission coefficient. In order to obtain the accuracy of 
RSSI value and reduce the error produced in the experiment, it is necessary to measure the size of $\mathrm{A}$ and $\mathrm{n}$. A and $\mathrm{n}$ are different in different experimental environment, in order to obtain accurate parameters $\mathrm{A}$ and $\mathrm{n}$, get accurate parameter information by linear regression analysis.

Assuming the position of the laboratory environment, the distance from $d_{i}$ to AP was measured as $\mathrm{RSSI}_{i}$, where $\mathrm{i}=1,2, \ldots \mathrm{n}$. Formula(2) can be obtained.

$$
n=\hat{n}=\frac{\sum_{i=1}^{n}\left(\rho_{i}-\bar{\rho}\right) R S S I_{i}}{\sum_{i=1}^{n}\left(\rho_{i}-\bar{\rho}\right)^{2}}
$$

$$
A=\overline{R S S I}-n \bar{\rho}
$$

$$
\text { Among them, } \bar{\rho}=\frac{1}{n} \rho_{\mathrm{\imath}} \overline{R S S I}=\frac{1}{n} \sum_{i=1}^{n} R_{S S I_{i}}
$$

In the experimental environment for testing, test sites from the AP location $1 \mathrm{~m}$ for 100 sets of experiments, to measure the RSSI value into the above formula can be obtained the values of $A$ and $n$, in this experiment, $A$ value is 39 and the $n$ value is 2

The detection process: first detection of AP's SSID, if the system is found to have the same SSID, and then compare the two AP's MAC. When have the same MAC address, then detect the value of RSSI. In the measurement of RSSI value, using the measured parameters $A=29$, the signal transmission coefficient $n=2$. When the RSSI value is greater than or less than the original value in the set threshold value between a, without warning, or generate warning messages, notify the administrator will be included in the AP phishing blacklist. As shown in figure 2.

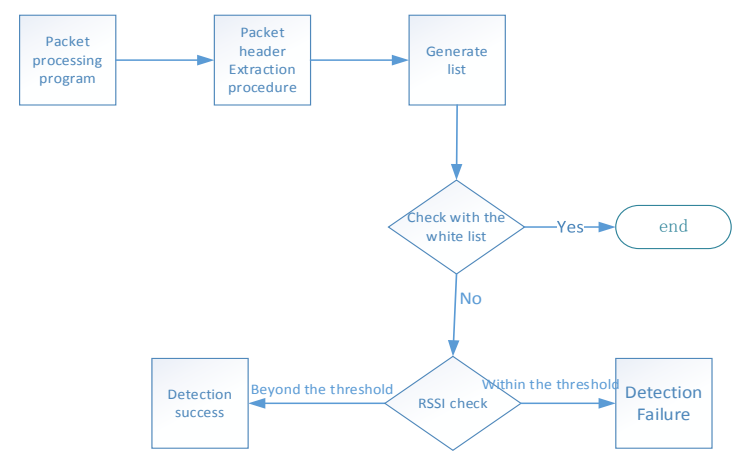

Fig. 2 detection process

For the RSSI value, at the same node, the RSSI value of the multi group was collected, and the relationship between the RSSI value and the measured distance was measured by linear regression analysis, as shown in Figure 3.

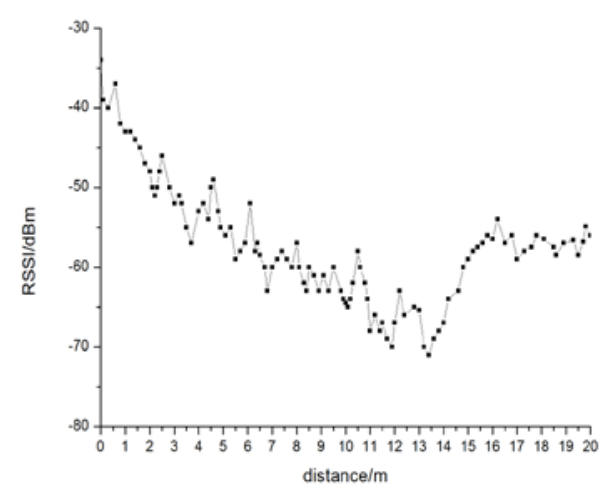

Fig. 3 RSSI values decay curve with distance

The scanner to detect the RSSI value size and data list in the corresponding AP RSSI values are compared, if make the absolute value of the difference in threshold within a corresponding AP that can not be detected this AP, if the difference is very big, can the AP detection is added to the black list. The threshold of a will be conducted in the subsequent experimental test sections.

\section{EXPERIMENT AND RESULT ANALYSIS}

In the scene, respectively, the two were detected with the same SSID AP detected two RSSI value, in order to determine the specific which fishing AP, on the threshold of a measure. In the experiment, detect the location of the fixed server and normal wireless AP, AP locations on the fishing change, measurement of normal wireless AP $\mathrm{RSSI}_{\mathrm{i}}$ and fishing AP $\mathrm{RSSI}_{\mathrm{j}}$. The threshold $\mathrm{a}=\left|R S S I_{i}-R S S I_{j}\right|$. Changing the fishing AP position, get the threshold a and the detection rate in a data from $1 \mathrm{~dB}$ to $6 \mathrm{~dB}$, were measured in 50 times. As shown in figure 4.

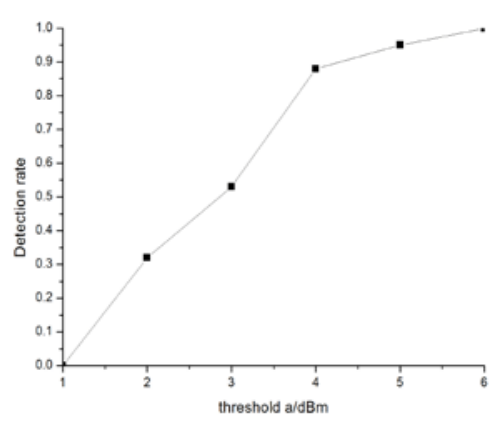

Fig.4 Detection Threshold

From Figure 4, we can get out, with a gradual increase of the threshold, the detection rate is gradually increased. When the threshold was $5 \mathrm{~dB}$, the detection rate was $95 \%$.

Next, the use of RSSI in the vicinity of the laboratory to detect AP, build the environment, using the specific API function to achieve. As shown in Table I, the information security laboratory, near the wireless network to get the results of the scan. 
Table I . Wireless Scan Results

\begin{tabular}{cccc}
\hline MAC Address & SSID & Channel & RSSI \\
\hline 0c:da:41:e9:99:81 & Gdut & 6 & -55 \\
8c:21:0a:c0:e1:86 & 514 & 1 & -53 \\
Ec:26:ca:77:6e:b8 & QQ & 6 & -51 \\
E4:d3:32:5e:ef:c0 & 732TK & 6 & -54 \\
C2:61:18:04:d7:c8 & ChinaNet & 6 & -54 \\
80:71:7a:42:1f:fc & Wd & 8 & -59 \\
5a:cf:5e:6c:0b:f6 & Rachel & 11 & -100 \\
0c:da:41:e9:d1:c0 & Gdut & 11 & -57 \\
6c:e8:73:a5:54:26 & 625-2s & 4 & -58 \\
Ec:26:ca:77:6e:b8 & QQ & 6 & -60 \\
C2:61:18:04:d7:c8 & Tkengine732732 & 6 & -54 \\
\hline
\end{tabular}

In the information security laboratory to build a phishing AP, its SSID as QQ, forged and normal legal AP consistent information, modify the soft MAC we build the AP address for the EC:26:CA:77:6E:B8, the communication channel is 6 .

We can clearly see the two AP SSID and the MAC address as the same, only by using the MAC address of the test, this failure. Next we use is presented in the design of the white list check, by white list database with the corresponding RSSI AP comparison, needs before comparing with the actual observation of an AP site location and changes, to prevent due to factors that change the legitimate AP location, leading to changes in the white list AP SSID, when position change needs promptly of any changes in the white list information. Compared to white list data, it is concluded that the specific RSSI values, both RSSI value difference is $9 \mathrm{~dB}$ is greater than threshold $5 \mathrm{~dB}$, so called illegal AP, namely RSSI value for -60 AP added to the blacklist, has now reached the detection.

\section{V.CONCLUSIONS}

In this paper, the principle of fishing AP attack in wireless LAN is analyzed, a method of AP detection based on RSSI is improved, and the condition of detecting fishing WIFI is analyzed. Through the use of the RSSI can not be forged and easy access to the characteristics, comprehensive use of the wireless MAC AP address information, to achieve the legitimacy of phishing AP detection. Experimental results show the effectiveness of this method.

The AP phishing detection method based on RSSI is in a server. In the RSSI difference within the range of $-5 \mathrm{dBm}$ can achieve $95 \%$ detection rate, and with the increase of the gap and the detection result is better, but when the two AP in less than $5 \mathrm{dBm}$, the detection effect and is not very ideal. In future work, will further study more general detection method, to investigate the attack methods in wireless network and can take advantage of the loopholes, for wireless network vulnerability analysis, proposed the corresponding preventive measures.

\section{ACKNOWLEDGEMENTS}

This work is supported by the science and technology project of Guangdong Province (No.2015B010128014, 2015B090906016,2014B090908011,2016B010107002,2016B 090918039,2016B090918125).

\section{REGERENCE}

[1] Fang Dingyi, Qi Shengde, Tang Yongzhan, Chen Xiaojiang, Gu Yuanxiang. A based on the RSSI of the smart home environment EvilTwin attack detection method [J]. Chinese Journal of computers, 2016,39:1-15

[2] Song Y, Yang C, Gu G. Who Is Peeping at Your Passwords at Starbucks? - To Catch an Evil Twin Access Point[C]// Dependable Systems and Networks, IEEE/IFIP International Conference on. IEEE, 2010:323 332

[3] Han H, Xu F, Tan C C, et al. Defending against vehicular rogue APs[C]// INFOCOM, 2011 Proceedings IEEE. IEEE, 2011:1665 - 1673.

[4] Han H, Sheng B, Tan C C, et al. A Timing-Based Scheme for Rogue AP Detection[J]. Parallel \& Distributed Systems IEEE Transactions on, 2011, 22(11):1912-1925.

[5] Han H, Xu F, Tan C C, et al. Defending against vehicular rogue APs[C]// INFOCOM, 2011 Proceedings IEEE. IEEE, 2011:1665 - 1673.

[6] Chen Wei, Gu Yang, Yu Le. Active phishing attacks and defense research in wireless networks with high concealment [J]. Journal of Wuhan University: Science Edition, 2013, 59 (2): 171-177.

[7] Chen Wei, Gu Yang, Li Chenyang, et al. Research on wireless fishing access point attack and detection technology [J]. Journal of Wuhan University: Science Edition, 2014, 60 (1): 13-23.

[8] Zhu Jianming, Ma Jianfeng. Wireless LAN Security: methods and techniques of $[\mathrm{M}]$. second edition. Beijing: Mechanical Industry Press, 2009.

[9] Bratus S, Cornelius C, Kotz D, et al. Active behavioral fingerprinting of wireless devices.[J]. In Proceedings of the First ACM Conference on Wireless Network Security WiSec, 2008:56-61.

[10] Jianghua, Ling Ling Ruan, Wang Xin. Based on SHA-256 message authentication in the four time handshake protocol [J]. Electronics and computer, 2014,08:155-158.

[11] Kim I, Seo J, Shon T, et al. A novel approach to detection of mobile rogue access points[J]. Security \& Communication Networks, 2014, 7(10):1510-1516. 